\title{
ORIGINAL
}

\section{Clinical markers and some trace elements in patients with type-2 diabetic nephropathy : Impact of insulin resistance}

\author{
Adnan Jassim Mohammed Al-Fartosy'), Nadhum Abdulnabi Awad'1), and Sadoun Abbas Alsalimi'1,2) \\ ${ }^{1)}$ Department of Chemistry, College of Science, University of Basrah, Basrah, Iraq, ${ }^{2}$ Department of Basic Medical Sciences, College of Nursing, \\ University of Basrah, Basrah, Iraq
}

\begin{abstract}
INTRODUCTION : Diabetic Nephropathy (DN) is the leading reason of all excess death-rate over type-2 diabetes mellitus (T2DM) patients with microalbuminuria, macroalbuminuria or end-stage renal disease. This work was aimed to estimate insulin resistance (IR) effect on some markers and trace elements (Selenium, Zinc, Magnesium) levels for early predicting of DN in T2DM patients. METHODS : We conducted a cross-sectional clinical study. Blood and urine samples were collected from 63 subjects with T2DM and 33 healthy controls to assess glucose, insulin, IR, urea, creatinine, glomerular filtration rate (GFR), creatinine clearance (CrCl), homocysteine (Hcy), fructosamine (FA), cystatin $\mathrm{C}(\mathrm{Cys})$, albumin (Alb), neutrophil gelatinase-associated lipocalin (NGAL), 8-hydroxy-2 -deoxyguanosine (8-OHdG) and trace elements levels. Data were collected using a questionnaire that was filled out by specialized doctors. RESULTS : Compared with controls, the results revealed that T2DM patients with or without DN had significant increases in glucose, insulin, IR, urea, creatinine, Hcy, FA, CysC, Alb, NGAL and 8-OHdG and significant decreases in GFR, $\mathrm{CrCl}$ and trace elements levels. Body mass index (BMI) had no changes. CONCLUSION : Uncontrolled T2DM was associated with BMI, IR and physical activity in which elevated Hcy, FA, CysC, Alb, NGAL and 8-OHdG levels and decreased trace elements levels may be used as early clinical markers of DN. J. Med. Invest. 68:76-84, February, 2021
\end{abstract}

Keywords: Diabetic nephropathy, Insulin resistance, Obesity, Oxidative stress, Trace elements

\section{INTRODUCTION}

The term of insulin resistance (IR) is elucidated the disability of cells response to the insulin action in transferring glucose from the bloodstream into muscle and tissues $(1,2)$. Diabetes mellitus (DM) is still an important health problem as rising numbers of patients with chronic and badly preserved diabetes develop diabetic nephropathy $(\mathrm{DN})(3,4)$.

$\mathrm{DN}$ is classically defined by the existence of proteinuria in the non-attendance of other renal disease. It is a common issue that is usually happen in patients who have hypertension, bad glycemic control, genetic predisposition or glomerular hyperfiltration (5). $\mathrm{DN}$ is may be representing a crucial cause of chronic kidney disease (CKD) that customarily leads to end-stage renal disease (ESRD) $(6,7)$. Urine albumin excretion (UAE) is deemed normal (normoalbuminuria) when it is less than $30 \mathrm{mg} /$ day (2-19 $\mu \mathrm{g} / \mathrm{mL}$ ). Also, UAE is classified as microalbuminuria (MAU) when it reaches $30-300 \mathrm{mg} /$ day, macroalbuminuria when it exceeds $300 \mathrm{mg} /$ day $(8,9)$.

Considering the raised happening of both DM and DN, the detection of early DN is fundamental importance to supply suitable therapy that prevents or slows evolution towards ESRD. Markers have a crucial role in the first detection of DN. These markers span period of normoalbuminuria that predates MAU but also development of renal involvement during MAU or macroalbuminuria (10).

Homocysteine (Hcy) is a homologue of the amino acid cysteine differing by an extra methylene (-CH2-) group. Hcy is not

Received for publication July 16, 2020 ; accepted November 10, 2020.

Address correspondence and reprint requests to Prof. Dr. Adnan Jassim Mohammed Al-Fartosy, Department of Chemistry, College of Science, University of Basrah, Basrah, Iraq. acquired from the food, but it is biosynthesized from methionine via a multi-step process. Hcy raised oxidant stress-triggered vascular dysfunction and the inverse impacts of Hcy on vascular function and neuroexcitation may participate in the developing and progress of dementia-type disorders (11).

Fructosamine (FA) is a compound resulting from the reaction between fructose and amine (with a molecule of water being released) and utilizing to recognize the plasma glucose level over time and assess diabetic control over an intermediate period since FA assessment exploits the capability of serum proteins to go through glycation (glycosylation) in situations of elevated ambient glycemia (12).

Cystatin $\mathrm{C}(\mathrm{CysC})$ is a low molecular weight protein produced at a stable rate by all nucleated cells. CysC is encoded by the CST3 gene and fundamentally used as a biomarker for kidney function and might play a role in brain disorder and an inhibitor of papain-like cysteine proteases. CysC has lately been shown inhibition to other family of cysteine protease called the peptidase family C13 with human legumain as a model enzyme (13).

Neutrophil Gelatinase-Associated Lipocalin (NGAL) is a small protein, almost $25 \mathrm{kD}$, that belongs to a superfamily of proteins called lipocalins which is a family from small proteins that cells send out to tie up things and convey them back and particularize in binding and carrying small hydrophobic molecules. NGAL possess varied functions like: motivating apoptosis, transferring, adjusting immune response and triggering nephrogenesis by inducing the diversion of mesenchymal cells to kidney epithelia. Also, NGAL plays a reno-protective function via promoting tubule cell proliferation in kidney injury particularly in ischemia-reperfusion injury (14).

8-Hydroxy-2 -deoxyguanosine (8-OHdG) is an output of oxidative DNA damage following specified enzymatic cleavage after reactive oxygen species (ROS) stimulated 8-hydroxylation of the guanine base in nuclear DNA and mitochondria. When impaired DNA is fixed, 8-OHdG is secreted in urine without 
further metabolism. 8-OHdG can be found in tissues, blood and urine of humans and it is considered an authoritative and key marker for cellular oxidative stress (15).

Although the scientific community has started resolving the secrets of the close linkage between IR, some blood and urine markers with trace elements and their physiological effects, a lot is still remaining to be discovered. In the province of Basrah (southern of Iraq), to date, no study has been investigated on IR, Hcy, FA, CysC, NGAL, 8-OHdG and trace elements (Selenium [Se], Zinc [Zn] and Magnesium [Mg]) and their effects on DN patients. Therefore, the present study is focused on assessing the IR effect on these markers and trace elements levels in DN patients in Basrah province-Iraq to obtain useful results that can be adopted in early diagnosis of $\mathrm{DN}$ in $\mathrm{T} 2 \mathrm{DM}$ patients.

\section{PATIENTS AND METHODS}

\section{Subjects}

The present study is a cross-sectional clinical experiment that investigated the IR impact on DN markers in T2DM subjects. Samples were collected from the subjects who were visiting "Diabetes and Endocrine Glands Centre" at Al-Mawany teaching hospital in Basrah province-Iraq, during the period from August 2019 until February 2020.

In this study, 63 subjects (men and women) who suffering from T2DM were selected to share in this study and they divided into two groups : the first group was 31 patients who suffering from DN (15 men and 16 women) while the second group was 32 patients who free from $\mathrm{DN}$ ( 15 men and 17 women). These subjects were matched with 33 healthy controls (16 men and 17 women).

Patients with the following inclusion criteria were shared in the current study ; age between $36-65$ years old ; T2DM patients (with or without $\mathrm{DN}$ ) were diagnosed by clinicians in the diabetes and endocrine glands centre in Al-Mawany teaching hospital. The control group was health individuals, not suffering from T2DM nor having any family history of T2DM, not suffering from any acute or chronic cardiovascular diseases (CVD's) and not taking any drugs believed to alter plasma glucose level. All the volunteers had a stable clinical course for at least 3 months.

Patients who were pregnant (women), had angina or heart failure, pancreatic diseases, hypertension, renal failure, severe liver dysfunction, alcoholics, T1DM, urinary tract infection, uncontrolled thyroid disorders, human immunodeficiency viruses (HIV) infections, hormonal abnormalities, genetic syndromes and severe concurrent illness were excluded from the study.

Diagnosis of T2DM patients was based on the recommendations of American Diabetes Association (16). DN was diagnosed based on GFR level $\left(<60 \mathrm{~mL} / \mathrm{min} / 1.73 \mathrm{~m}^{2}\right)$ or persistent microalbuminuria ( $>19 \mu \mathrm{g} / \mathrm{mL}$ ) in the morning urine of patients (17, 18). Demographical data were collected via a structural interview that was conducted during the visit. Standard self-administered questionnaire paper is used to define the age, duration of $\mathrm{DM}$ and DN, health habits (smoking, alcohol consumption and exercise), medical history and current medications.

\section{Sampling procedures}

All samples were collected in the morning between 09:0010:00 AM after 12 hours fasting time and 30 minutes of rest in the supine position.

Fresh venous blood $(10 \mathrm{~mL})$ was collected from all volunteers by vein punch then divided into two parts : the first part $(1 \mathrm{~mL})$ was added into special tube with anticoagulant and shook gently as a whole blood to utilize for Se level determination. The second part $(9 \mathrm{~mL})$ was moved to plain tube (without anticoagulant) which admitted clotting for 20 minutes at room temperature.
Then, it was moved into a centrifuge at $402 \times \mathrm{g}$ for 20 minutes to get the serum.

Also, Fresh morning urine $(20 \mathrm{~mL})$ was collected from all volunteers in pre-sterile vials, after first voids of urine, the sample was centrifuged at $402 \times \mathrm{g}$ for 20 minutes to remove all suspended particles. Then, the clear supernatant was collected carefully.

All the collected samples (whole blood, serum and urine) were immediately utilized in the estimation of the variables in this study, while the rest were stored in deep freezing at $\left(-80^{\circ} \mathrm{C}\right)$ until using.

\section{Clinical and laboratory measurements}

Serum glucose, urea and creatinine were determined by UVVis Spectrophotometer (UV-EMC-LAB, Duisburg, Germany) and using the following kits (Randox, County Antrim, UK, Cat. No. : GL364 ; Linear, Barcelona, Spain, Cat. No. : 1156015; Randox, County Antrim, UK, Cat. No. : CR 511/S) respectively.

Serum (insulin, Hcy, FA and CysC) and urine (Alb, NGAL and 8-OHdG) markers were assayed by human ELISA kits (Calbiotech, California, USA, Cat. No. : IS130D ; BT-Lab, Shanghai, China, Cat. No. : E3292Hu ; BT-Lab, Shanghai, China, Cat. No. : E3232Hu ; BT-Lab, Shanghai, China, Cat. No. : E1104Hu ; Creative Diagnostics, New York, USA, Cat. No. : DEIA2299 ; BTLab, Shanghai, China, Cat. No. : E1719Hu ; BT-Lab, Shanghai, China, Cat. No. : EA0048Hu), respectively. Sandwich ELISA technique was applied, and the level of each biomarker was assayed using a standard curve.

\section{Blood trace elements estimation}

Serum $\mathrm{Zn}$ and $\mathrm{Mg}$ were determined by using GBC 933 Plus flame atomic absorption spectrometry "FAAS" (GBC, Braeside, Australia) (19).

While, whole blood Se was determined by Flame Atomic Absorption Spectrometer with Homemade Hydride Generation System (Shimadzu, Tokyo, Japan) (20).

\section{Clinical parameters calculations}

Body mass index (BMI) was calculated by the standard BMI equation (21) :

$\operatorname{BMI}\left(\mathrm{kg} / \mathrm{m}^{2}\right)=$ weight $(\mathrm{kg}) /$ height $\left(\mathrm{m}^{2}\right)$

IR was calculated by the Homeostatic Model Assessment for Insulin Resistance (HOMA-IR) equation (22) :

HOMA-IR $=$ Fasting insulin $(\mu \mathrm{IU} / \mathrm{mL}) \times$ Fasting glucose $(\mathrm{mg} / \mathrm{dL}) / 405$

Glomerular filtration rate (GFR) was calculated by the Modification of Diet in Renal Disease Study (MDRD) equation (23) :

GFR $\left(\mathrm{mL} / \mathrm{min} / 1.73 \mathrm{~m}^{2}\right)=186 \times$ Serum $\mathrm{Cr}^{-1.154} \times$ age $^{-0.203} \times$ 1.212 (if subject is black) $\times 0.742$ (if subject is female)

Creatinine clearance $(\mathrm{CrCl})$ was calculated by the Cockcroft-Gault equation (23) :

$\mathrm{CrCl}(\mathrm{mL} / \mathrm{min})=(140-$ age $) \times($ weight in $\mathrm{kg}) \times(0.85$ if female) / $(72 \times$ Serum Cr $)$

\section{Statistical Analysis}

Statistical analysis of data was carried out using statistical package for the social sciences (SPSS) and the values expressed as mean \pm SD. The values of $p>0.05$ was to be considered statistically not significant, $\mathrm{p}<0.05$ was considered as significant differences and $\mathrm{p}<0.01$ for highly significant.

\section{Ethical considerations}

This study was conducted in accordance with the Declaration of Helsinki and the protocol of this study was approved by the Ethics and Behavioural Research Committee (Scientific Committee) in the Department of Chemistry - College of Science University of Basrah - Basrah - Iraq. The procedures were fully 
clarified to all subjects and an informed consent form was signed and obtained from all the participants.

\section{RESULTS}

The general demographic characteristics of all volunteers participated in this work were presented in Table 1.

As compared to healthy controls, the results revealed that T2DM patients with or without DN had a non-significant change $(p>0.05)$ in BMI level, as shown in Table 2.

On the other hand, data obtained in our study (in Tables 2 and 4) indicated that T2DM patients with or without DN had a high significant $(p<0.01)$ increase in levels of serum (glucose, insulin, HOMA-IR, Hcy and FA) and urine (NGAL and 8-OHdG) markers as compared to healthy group.

Furthermore, T2DM patients with DN had a high significant increase $(p<0.01)$ and patients with T2DM without DN had a significant increase $(p<0.05)$ in the levels of serum urea, creatinine, CysC and urinary Alb as compared to controls, as demonstrated in Tables 2 and 4.

Moreover, our data reported that GFR and $\mathrm{CrCl}$ levels were decreased significantly in T2DM patients $(p<0.01$ in patients with $\mathrm{DN}$ and $\mathrm{p}<0.05$ in patients without $\mathrm{DN}$ ) as compared to controls, as illustrated in Table 2 .

Finally, results in Table 3 revealed that the levels of blood trace elements (Se, Zn and $\mathrm{Mg}$ ) were highly significantly $(\mathrm{p}<0.01)$ declined in T2DM patients with and without DN as compared to controls.

\section{DISCUSSION}

In the current study, data presented that all the patients and healthy control subjects were non-smokers. Also, most of the volunteers from both patients and healthy control were from urban, all of them acquired a well-educated and they had a good work place. The major variations between populations of urban and rural regions are the differences in food habits, genetic, social, psychic, pollution, environments and others which are raising dramatically in urban areas (22).

Obesity may be representing a diabetogenic factor via elevating resistance to the action of insulin in those genetically predisposed to enhance T2DM. So, it could be acts as one of the etiological factors in the development of T2DM, and mostly because of loss of early phase insulin secretion in response to glucose which happens relatively earlier in the development of T2DM (24). This loss is critically crucial as the early blast of insulin secretion plays a substantial role in priming target tissues of insulin, especially the liver responsible for normal glucose homeostasis after food uptake and mealtime glucose deflection take place when this process was deteriorated (25). Furthermore, it may consider one of the modifiable cardiovascular risk factors that is far more predominant in those people with T2DM than in the general population. Moreover, obesity and physical inactivity are important independent risk factors for T2DM in middle aged of both men and women (26).

Insulin resistance (IR) can be known as a form of biological misinformation in the body in which the insulin hormone receptors on the cell membrane are not responding suitably to insulin. Thus, the glucose in blood becomes unable to reach into cells which could lead to a hypoglycaemic reaction. This condition makes the pancreas produces high doses of insulin to endeavour getting the glucose out of blood into cells. So, this leads to decrease in the ability of insulin to adjust and signal changes in the levels of glucose in the blood and perhaps grows IR (21). HOMA-IR was used as a substitute measure of IR in our study. Although HOMA-IR was not the gold standard for assessment of insulin sensitivity, but it was a clinically useful index in many studies (27). The function of $\beta$-cells may be damaged during adolescence or later. Its deterioration ranges from hyperinsulinemia, secondary to IR, with normal glucose tolerance to $\beta$-cells failure with T1DM. Furthermore, when insulin secretion is decreased (IR is present) in the later stages of the

Table 1. The demographic characteristics of the present study.

\begin{tabular}{|c|c|c|c|c|}
\hline \multirow{2}{*}{\multicolumn{2}{|c|}{ The Characteristics }} & \multicolumn{2}{|c|}{ T2DM Patients } & \multirow{2}{*}{ Healthy control } \\
\hline & & with DN & without DN & \\
\hline \multicolumn{2}{|c|}{ Total subjects No. } & 31 & 32 & 33 \\
\hline \multicolumn{2}{|c|}{ Age (Years) (Mean \pm SD) } & $57.26 \pm 4.47$ & $53.94 \pm 4.74$ & $51.70 \pm 5.07$ \\
\hline \multicolumn{2}{|c|}{ DM duration (Years) (Mean \pm SD) } & $13.57 \pm 2.53$ & $5.23 \pm 1.66$ & - \\
\hline \multicolumn{2}{|c|}{$\mathrm{DN}$ duration (Years) (Mean $\pm \mathrm{SD})$} & $6.80 \pm 0.63$ & - & - \\
\hline \multirow{2}{*}{$\begin{array}{c}\text { Demographic } \\
\text { area }\end{array}$} & Urban & 28 & 27 & 29 \\
\hline & Rural & 3 & 5 & 4 \\
\hline \multirow{2}{*}{$\begin{array}{l}\text { Educational } \\
\text { background }\end{array}$} & Learned & 25 & 24 & 26 \\
\hline & Illiterate & 6 & 8 & 7 \\
\hline \multirow{2}{*}{$\begin{array}{l}\text { Smoking } \\
\text { habits }\end{array}$} & Positive & 0 & 0 & 0 \\
\hline & Negative & 31 & 32 & 33 \\
\hline \multirow{2}{*}{$\begin{array}{l}\text { Food } \\
\text { habits }\end{array}$} & Vegetarian & 5 & 6 & 8 \\
\hline & Non-vegetarian & 26 & 26 & 25 \\
\hline \multirow{2}{*}{$\begin{array}{l}\text { Employment } \\
\text { status }\end{array}$} & Employed & 19 & 14 & 28 \\
\hline & Not employed & 12 & 18 & 5 \\
\hline \multirow{2}{*}{ Gender } & Men & 15 & 15 & 16 \\
\hline & Women & 16 & 17 & 17 \\
\hline
\end{tabular}


The Journal of Medical Investigation Vol. 68 February 2021

Table 2. Levels of serum markers in men and women of healthy control and T2DM patients with and without DN.

\begin{tabular}{|c|c|c|c|c|c|c|c|c|c|c|c|c|}
\hline \multirow{4}{*}{ Marker } & \multirow{4}{*}{ Gender } & \multicolumn{10}{|c|}{ T2DM Patients } & \multirow{4}{*}{$\begin{array}{c}\begin{array}{c}\text { Healthy } \\
\text { Control }\end{array} \\
\begin{array}{c}\text { Mean } \pm \\
\text { SD }\end{array}\end{array}$} \\
\hline & & & & with DN & & & & & thout DI & & & \\
\hline & & \multirow{2}{*}{$\begin{array}{l}\text { Mean } \pm \\
\text { SD }\end{array}$} & \multirow{2}{*}{$\mathrm{SE}$} & \multirow{2}{*}{ Range } & \multicolumn{2}{|c|}{$95 \% \mathrm{CI}$} & \multirow{2}{*}{$\begin{array}{c}\text { Mean } \pm \\
\text { SD }\end{array}$} & \multirow{2}{*}{$\mathrm{SE}$} & \multirow{2}{*}{ Range } & \multicolumn{2}{|c|}{$95 \% \mathrm{CI}$} & \\
\hline & & & & & Lower & Upper & & & & Lower & Upper & \\
\hline \multirow{2}{*}{$\begin{array}{c}\mathrm{BMI} \\
\left(\mathrm{Kg} / \mathrm{m}^{2}\right)\end{array}$} & Men & $\begin{array}{c}31.27 \pm \\
1.67\end{array}$ & 0.43 & $\begin{array}{c}28.7- \\
34.1\end{array}$ & 26.64 & 35.91 & $\begin{array}{c}30.30 \pm \\
2.56\end{array}$ & 0.66 & $\begin{array}{c}26.3- \\
34.6\end{array}$ & 23.19 & 37.41 & $\begin{array}{c}31.56 \pm \\
0.25\end{array}$ \\
\hline & Women & $\begin{array}{c}30.12 \pm \\
0.51\end{array}$ & 0.13 & $\begin{array}{c}29.8- \\
32.1\end{array}$ & 28.70 & 31.53 & $\begin{array}{c}30.86 \pm \\
0.38\end{array}$ & 0.09 & $\begin{array}{c}29.6- \\
31\end{array}$ & 29.81 & 31.92 & $\begin{array}{c}30.08 \pm \\
1.74\end{array}$ \\
\hline \multirow{2}{*}{$\begin{array}{l}\text { Glucose } \\
(\mathrm{mg} / \mathrm{dL})\end{array}$} & Men & $\begin{array}{c}172.73 \pm \\
3.35 * *\end{array}$ & 0.86 & $\begin{array}{c}162- \\
180\end{array}$ & 163.43 & 182.03 & $\begin{array}{c}168.60 \pm \\
3.09 * *\end{array}$ & 0.80 & $\begin{array}{l}158- \\
174\end{array}$ & 160.02 & 177.18 & $\begin{array}{c}104.26 \pm \\
3.82\end{array}$ \\
\hline & Women & $\begin{array}{c}162.38 \pm \\
2.34 * * \\
\end{array}$ & 0.59 & $\begin{array}{c}159- \\
171 \\
\end{array}$ & 155.88 & 168.87 & \begin{tabular}{|c|}
$158.76 \pm$ \\
$2.15^{* *}$ \\
\end{tabular} & 0.52 & $\begin{array}{c}151- \\
163\end{array}$ & 152.80 & 164.73 & $\begin{array}{c}99.68 \pm \\
4.56 \\
\end{array}$ \\
\hline \multirow{2}{*}{$\begin{array}{c}\text { Insulin } \\
(\mu \mathrm{U} / \mathrm{mL})\end{array}$} & Men & $\begin{array}{l}29.06 \pm \\
2.72 * *\end{array}$ & 0.70 & $\begin{array}{c}24.65- \\
33.47\end{array}$ & 21.51 & 36.61 & $\begin{array}{l}28.17 \pm \\
2.37 * * \\
\end{array}$ & 0.61 & $\begin{array}{c}24.32- \\
32.02\end{array}$ & 21.59 & 34.75 & $\begin{array}{c}11.50 \pm \\
1.81\end{array}$ \\
\hline & Women & $\begin{array}{l}28.78 \pm \\
1.14 * *\end{array}$ & 0.29 & $\begin{array}{c}26.84- \\
30.62\end{array}$ & 25.62 & 31.95 & $\begin{array}{l}27.99 \pm \\
1.88 * *\end{array}$ & 0.46 & $\begin{array}{c}24.69- \\
31.13\end{array}$ & 22.77 & 33.21 & $\begin{array}{c}10.70 \pm \\
1.17\end{array}$ \\
\hline \multirow{2}{*}{ HOMA-IR } & Men & $\begin{array}{l}12.41 \pm \\
1.31 * * \\
\end{array}$ & 0.34 & $\begin{array}{l}9.9- \\
14.9\end{array}$ & 8.78 & 16.05 & $\begin{array}{l}11.74 \pm \\
1.11 * *\end{array}$ & 0.29 & $\begin{array}{l}9.5- \\
13.8 \\
\end{array}$ & 8.66 & 14.82 & $\begin{array}{c}2.98 \pm \\
0.58\end{array}$ \\
\hline & Women & $\begin{array}{l}11.54 \pm \\
0.55 * *\end{array}$ & 0.14 & $\begin{array}{c}10.5- \\
12.9\end{array}$ & 10.01 & 13.06 & $\begin{array}{l}10.98 \pm \\
0.82 * *\end{array}$ & 0.20 & $\begin{array}{l}9.2- \\
12.5 \\
\end{array}$ & 8.70 & 13.25 & $\begin{array}{c}2.64 \pm \\
0.41\end{array}$ \\
\hline \multirow{2}{*}{$\begin{array}{c}\text { Urea } \\
(\mathrm{mg} / \mathrm{dL})\end{array}$} & Men & $\begin{array}{l}59.31 \pm \\
3.97 * * \\
\end{array}$ & 1.03 & $\begin{array}{l}52.87- \\
65.75\end{array}$ & 48.29 & 70.33 & $\begin{array}{c}36.70 \pm \\
2.59^{*}\end{array}$ & 0.67 & $\begin{array}{c}32.5- \\
40.9\end{array}$ & 29.51 & 43.89 & $\begin{array}{c}29.63 \pm \\
4.05\end{array}$ \\
\hline & Women & $\begin{array}{l}53.94 \pm \\
1.80 * *\end{array}$ & 0.45 & $\begin{array}{l}51.01- \\
56.86\end{array}$ & 48.94 & 58.93 & $\begin{array}{c}30.12 \pm \\
2.11 *\end{array}$ & 0.51 & $\begin{array}{l}24.9- \\
37.09\end{array}$ & 24.26 & 35.97 & $\begin{array}{c}25.04 \pm \\
2.28\end{array}$ \\
\hline \multirow{2}{*}{$\begin{array}{l}\text { Creatinine } \\
(\mathrm{mg} / \mathrm{dL})\end{array}$} & Men & $\begin{array}{c}1.77 \pm \\
0.19 * *\end{array}$ & 0.05 & $\begin{array}{l}1.38- \\
1.96\end{array}$ & 1.24 & 2.30 & $\begin{array}{l}1.15 \pm \\
0.08 *\end{array}$ & 0.02 & $\begin{array}{c}1.01- \\
1.29\end{array}$ & 0.93 & 1.37 & $\begin{array}{l}0.93 \pm \\
0.05\end{array}$ \\
\hline & Women & $\begin{array}{c}1.43 \pm \\
0.05^{* *}\end{array}$ & 0.01 & $\begin{array}{l}1.35- \\
1.50\end{array}$ & 1.29 & 1.56 & $\begin{array}{l}0.97 \pm \\
0.05 *\end{array}$ & 0.01 & $\begin{array}{c}0.89- \\
1.05\end{array}$ & 0.83 & 1.11 & $\begin{array}{l}0.81 \pm \\
0.05\end{array}$ \\
\hline \multirow{2}{*}{$\begin{array}{c}\text { GFR } \\
\left(\mathrm{mL} / \mathrm{min} / 1.73 \mathrm{~m}^{2}\right)\end{array}$} & Men & $\begin{array}{l}43.24 \pm \\
6.92 * *\end{array}$ & 1.79 & $\begin{array}{c}36.8- \\
58\end{array}$ & 24.03 & 62.45 & $\begin{array}{l}70.83 \pm \\
7.32 *\end{array}$ & 1.89 & $\begin{array}{l}60- \\
83.8\end{array}$ & 50.51 & 91.15 & $\begin{array}{c}91.19_{ \pm} \\
6.86\end{array}$ \\
\hline & Women & $\begin{array}{l}40.40 \pm \\
2.17 * * \\
\end{array}$ & 0.54 & $\begin{array}{l}37- \\
44.1\end{array}$ & 34.38 & 46.42 & \begin{tabular}{|c|}
$64.28 \pm$ \\
$4.91 *$ \\
\end{tabular} & 1.19 & $\begin{array}{c}56.6- \\
72.9\end{array}$ & 50.65 & 77.91 & $\begin{array}{c}80.11 \pm \\
7.22 \\
\end{array}$ \\
\hline \multirow{2}{*}{$\begin{array}{c}\mathrm{CrCl} \\
(\mathrm{mL} / \mathrm{min})\end{array}$} & Men & $\begin{array}{l}66.53 \pm \\
8.83 * *\end{array}$ & 2.28 & $\begin{array}{l}58- \\
85\end{array}$ & 42.02 & 91.05 & $\begin{array}{l}96.07 \pm \\
10.91 *\end{array}$ & 2.82 & $\begin{array}{l}81- \\
113\end{array}$ & 65.78 & 126.35 & $\begin{array}{c}130.31 \pm \\
12.13\end{array}$ \\
\hline & Women & $\begin{array}{l}62.88 \pm \\
5.31 * *\end{array}$ & 1.33 & $\begin{array}{c}54- \\
71\end{array}$ & 48.13 & 77.62 & $\begin{array}{c}99.76 \pm \\
9.71^{*}\end{array}$ & 2.36 & $\begin{array}{l}85- \\
116\end{array}$ & 72.81 & 126.72 & $\begin{array}{c}126.06 \pm \\
8.06\end{array}$ \\
\hline \multirow{2}{*}{$\begin{array}{c}\text { Hcy } \\
(\mathrm{nmol} / \mathrm{mL})\end{array}$} & Men & $\begin{array}{l}23.26 \pm \\
2.77 * *\end{array}$ & 0.72 & $\begin{array}{c}20.76- \\
29.02\end{array}$ & 15.57 & 30.95 & $\begin{array}{l}18.27 \pm \\
3.97 * *\end{array}$ & 1.03 & $\begin{array}{c}11.83- \\
24.71\end{array}$ & 7.25 & 29.29 & $\begin{array}{c}10.89 \pm \\
2.21\end{array}$ \\
\hline & Women & $\begin{array}{l}19.05 \pm \\
1.61 * *\end{array}$ & 0.40 & $\begin{array}{c}14.73- \\
23.81\end{array}$ & 14.58 & 23.52 & $\begin{array}{l}17.62 \pm \\
3.87 * *\end{array}$ & 0.94 & $\begin{array}{l}11.3- \\
23.94\end{array}$ & 6.88 & 28.36 & $\begin{array}{c}10.78 \pm \\
2.19\end{array}$ \\
\hline \multirow{2}{*}{$\underset{(\mathrm{mmol} / \mathrm{L})}{\mathrm{FA}}$} & Men & $\begin{array}{c}0.47 \pm \\
0.15^{* *}\end{array}$ & 0.04 & $\begin{array}{c}0.33- \\
0.97\end{array}$ & 0.06 & 0.89 & $\begin{array}{c}0.36 \pm \\
0.08^{* *}\end{array}$ & 0.02 & $\begin{array}{c}0.22- \\
0.50\end{array}$ & 0.14 & 0.58 & $\begin{array}{c}0.26 \pm \\
0.03\end{array}$ \\
\hline & Women & $\begin{array}{c}0.42 \pm \\
0.07 * * \\
\end{array}$ & 0.02 & $\begin{array}{l}0.32- \\
0.55\end{array}$ & 0.22 & 0.61 & $\begin{array}{c}0.34 \pm \\
0.07^{* *} \\
\end{array}$ & 0.02 & $\begin{array}{c}0.22- \\
0.46 \\
\end{array}$ & 0.15 & 0.54 & $\begin{array}{c}0.25 \pm \\
0.04\end{array}$ \\
\hline \multirow{2}{*}{$\begin{array}{c}\text { CysC } \\
(\mathrm{ng} / \mathrm{mL})\end{array}$} & Men & $\begin{array}{c}1.36 \pm \\
0.35 \text { ** }\end{array}$ & 0.09 & $\begin{array}{c}0.89- \\
2.39\end{array}$ & 0.39 & 2.33 & $\begin{array}{l}1.25 \pm \\
0.26 *\end{array}$ & 0.07 & $\begin{array}{c}0.95- \\
1.75\end{array}$ & 0.53 & 1.97 & $\begin{array}{c}0.79 \pm \\
0.07\end{array}$ \\
\hline & Women & $\begin{array}{c}1.28 \pm \\
0.28^{* *}\end{array}$ & 0.07 & $\begin{array}{c}0.91- \\
1.81\end{array}$ & 0.50 & 2.05 & $\begin{array}{l}1.21 \pm \\
0.21^{*}\end{array}$ & 0.05 & $\begin{array}{c}1.01- \\
1.67\end{array}$ & 0.62 & 1.79 & $\begin{array}{c}0.74 \pm \\
0.16\end{array}$ \\
\hline
\end{tabular}

Data are presented as mean \pm standard deviation (SD) ; SE : Standard error, Range : is the difference between the highest and lowest values in the set, $95 \% \mathrm{CI}$ : Confidence intervals (Lower and Upper) ; significance level is indicated by *, Where: $p>0.05: p$-value not significant, * indicates $\mathrm{p}<0.05$ (p-value significant) $; * *$ indicates $\mathrm{p}<0.01$ (p-value highly significant), in comparison with the corresponding control value. 
Table 3. Levels of trace elements in blood in men and women of healthy control and T2DM patients with and without DN.

\begin{tabular}{|c|c|c|c|c|c|c|c|c|c|c|c|c|}
\hline \multirow{4}{*}{ Biomarker } & \multirow{4}{*}{ Gender } & \multicolumn{10}{|c|}{ T2DM Patients } & \multirow{4}{*}{$\begin{array}{c}\begin{array}{c}\text { Healthy } \\
\text { Control }\end{array} \\
\begin{array}{c}\text { Mean } \pm \\
\text { SD }\end{array}\end{array}$} \\
\hline & & \multicolumn{5}{|c|}{ with DN } & \multicolumn{5}{|c|}{ without DN } & \\
\hline & & \multirow{2}{*}{$\begin{array}{c}\text { Mean } \pm \\
\text { SD }\end{array}$} & \multirow{2}{*}{$\mathrm{SE}$} & \multirow{2}{*}{ Range } & \multicolumn{2}{|c|}{$95 \% \mathrm{CI}$} & \multirow{2}{*}{$\begin{array}{l}\text { Mean } \pm \\
\text { SD }\end{array}$} & \multirow{2}{*}{$\mathrm{SE}$} & \multirow{2}{*}{ Range } & \multicolumn{2}{|c|}{$95 \% \mathrm{CI}$} & \\
\hline & & & & & Lower & Upper & & & & Lower & Upper & \\
\hline \multirow{2}{*}{$\begin{array}{c}\mathrm{Se} \\
(\mathrm{ng} / \mathrm{mL})\end{array}$} & Men & $\begin{array}{l}63.59 \pm \\
3.41 * *\end{array}$ & 0.88 & $\begin{array}{l}62.12- \\
67.65\end{array}$ & 54.13 & 73.06 & $\begin{array}{l}70.92 \pm \\
1.85 * *\end{array}$ & 0.48 & $\begin{array}{c}69.34- \\
73.64\end{array}$ & 65.78 & 76.05 & $\begin{array}{c}92.81 \pm \\
1.08 \\
\end{array}$ \\
\hline & Women & $\begin{array}{l}50.88 \pm \\
1.95 * *\end{array}$ & 0.49 & $\begin{array}{l}49.27- \\
55.08\end{array}$ & 45.46 & 56.29 & $\begin{array}{l}59.53 \pm \\
1.72 * *\end{array}$ & 0.42 & $\begin{array}{c}58.47- \\
64.21\end{array}$ & 54.76 & 64.31 & $\begin{array}{c}80.14 \pm \\
3.29 \\
\end{array}$ \\
\hline \multirow{2}{*}{$\underset{(\mu \mathrm{g} / \mathrm{mL})}{\mathrm{Zn}}$} & Men & $\begin{array}{c}0.80 \pm \\
0.26^{* *}\end{array}$ & 0.07 & $\begin{array}{c}0.50- \\
1.31\end{array}$ & 0.08 & 1.52 & $\begin{array}{c}0.96 \pm \\
0.16^{* *}\end{array}$ & 0.04 & $\begin{array}{c}0.55- \\
1.25\end{array}$ & 0.52 & 1.40 & $\begin{array}{c}1.59 \pm \\
0.30\end{array}$ \\
\hline & Women & $\begin{array}{c}0.70 \pm \\
0.22^{* *}\end{array}$ & 0.06 & $\begin{array}{c}0.42- \\
1.12\end{array}$ & 0.09 & 1.32 & $\begin{array}{c}0.77 \pm \\
0.18^{* *}\end{array}$ & 0.04 & $\begin{array}{c}0.53- \\
1.13 \\
\end{array}$ & 0.27 & 1.27 & $\begin{array}{l}1.01 \pm \\
0.32 \\
\end{array}$ \\
\hline \multirow{2}{*}{$\underset{(\mu \mathrm{g} / \mathrm{mL})}{\mathrm{Mg}}$} & Men & $\begin{array}{l}16.71 \pm \\
1.38^{* *}\end{array}$ & 0.36 & $\begin{array}{c}14.47- \\
18.95\end{array}$ & 12.88 & 20.54 & $\begin{array}{l}19.93 \pm \\
1.12 * *\end{array}$ & 0.29 & $\begin{array}{l}18.11- \\
21.75\end{array}$ & 16.82 & 23.04 & $\begin{array}{c}23.42 \pm \\
3.78 \\
\end{array}$ \\
\hline & Women & $\begin{array}{l}12.95 \pm \\
2.51 * *\end{array}$ & 0.63 & $\begin{array}{l}8.71- \\
16.97\end{array}$ & 5.98 & 19.92 & $\begin{array}{l}14.94 \pm \\
1.43 * *\end{array}$ & 0.35 & $\begin{array}{c}12.43- \\
17.33\end{array}$ & 10.97 & 18.91 & $\begin{array}{c}20.15 \pm \\
2.68\end{array}$ \\
\hline
\end{tabular}

Data are presented as mean \pm standard deviation (SD) ; SE : Standard error, Range : is the difference between the highest and lowest values in the set, $95 \% \mathrm{CI}$ : Confidence intervals (Lower and Upper); significance level is indicated by *, Where : $p>0.05: p$-value not significant, * indicates $\mathrm{p}<0.05$ ( $\mathrm{p}$-value significant) $; * *$ indicates $\mathrm{p}<0.01$ (p-value highly significant), in comparison with the corresponding control value.

Table 4. Levels of urine markers in men and women of healthy control and T2DM patients with and without DN.

\begin{tabular}{|c|c|c|c|c|c|c|c|c|c|c|c|c|}
\hline \multirow{4}{*}{ Biomarker } & \multirow{4}{*}{ Gender } & \multicolumn{10}{|c|}{ T2DM Patients } & \multirow{4}{*}{$\begin{array}{c}\begin{array}{c}\text { Healthy } \\
\text { Control }\end{array} \\
\begin{array}{c}\text { Mean } \pm \\
\text { SD }\end{array}\end{array}$} \\
\hline & & \multirow{3}{*}{$\begin{array}{l}\text { Mean } \pm \\
\text { SD }\end{array}$} & \multicolumn{4}{|c|}{ with DN } & \multicolumn{5}{|c|}{ without DN } & \\
\hline & & & & Dopse & \multicolumn{2}{|c|}{$95 \% \mathrm{CI}$} & \multirow{2}{*}{$\begin{array}{l}\text { Mean } \pm \\
\text { SD }\end{array}$} & \multirow{2}{*}{$\mathrm{SE}$} & \multirow{2}{*}{ Range } & \multicolumn{2}{|c|}{$95 \% \mathrm{CI}$} & \\
\hline & & & $\mathrm{SE}$ & Range & Lower & Upper & & & & Lower & Upper & \\
\hline \multirow{2}{*}{$\begin{array}{c}\mathrm{Alb} \\
(\mu \mathrm{g} / \mathrm{mL})\end{array}$} & Men & $\begin{array}{l}29.58 \pm \\
4.62 * *\end{array}$ & 1.19 & $\begin{array}{c}22.09- \\
37.07\end{array}$ & 16.75 & 42.41 & $\begin{array}{l}11.18 \pm \\
3.11^{*}\end{array}$ & 0.80 & $\begin{array}{l}6.14- \\
16.22 \\
\end{array}$ & 2.55 & 19.81 & $\begin{array}{l}6.17 \pm \\
2.13\end{array}$ \\
\hline & Women & $\begin{array}{l}28.54 \pm \\
5.25^{* *}\end{array}$ & 1.31 & $\begin{array}{c}19.99- \\
37.09\end{array}$ & 13.97 & 43.11 & $\begin{array}{c}10.68 \pm \\
2.59 *\end{array}$ & 0.63 & $\begin{array}{l}6.44- \\
14.92\end{array}$ & 3.49 & 17.87 & $\begin{array}{l}5.84 \pm \\
2.06\end{array}$ \\
\hline \multirow{2}{*}{$\begin{array}{l}\text { NGAL } \\
(\mathrm{ng} / \mathrm{mL})\end{array}$} & Men & $\begin{array}{l}55.26 \pm \\
2.51^{* *} \\
\end{array}$ & 0.65 & $\begin{array}{c}53.32- \\
60.60\end{array}$ & 48.29 & 62.23 & $\begin{array}{l}46.38 \pm \\
4.37 * *\end{array}$ & 1.13 & $\begin{array}{c}39.35- \\
53.87\end{array}$ & 34.25 & 58.52 & $\begin{array}{c}14.78 \pm \\
2.67\end{array}$ \\
\hline & Women & $\begin{array}{l}77.42 \pm \\
5.53^{* *}\end{array}$ & 1.38 & $\begin{array}{l}68.42- \\
86.49\end{array}$ & 62.07 & 92.78 & $\begin{array}{l}72.09 \pm \\
4.82 * *\end{array}$ & 1.17 & $\begin{array}{c}64.87- \\
80.04\end{array}$ & 58.71 & 85.47 & $\begin{array}{c}35.73 \pm \\
4.11\end{array}$ \\
\hline \multirow{2}{*}{$\begin{array}{l}\text { 8-OHdG } \\
(\mathrm{ng} / \mathrm{mL})\end{array}$} & Men & $\begin{array}{l}26.92 \pm \\
4.45^{* *}\end{array}$ & 1.15 & $\begin{array}{c}19.71- \\
34.13\end{array}$ & 14.57 & 39.27 & $\begin{array}{l}21.43 \pm \\
4.43^{* *}\end{array}$ & 1.14 & $\begin{array}{c}14.25- \\
28.61\end{array}$ & 9.13 & 33.73 & $\begin{array}{c}13.41 \pm \\
3.13\end{array}$ \\
\hline & Women & $\begin{array}{l}24.47 \pm \\
5.76^{* *}\end{array}$ & 1.44 & $\begin{array}{c}15.09- \\
33.84\end{array}$ & 8.48 & 40.45 & $\begin{array}{l}21.09 \pm \\
6.76 * *\end{array}$ & 1.64 & $\begin{array}{c}10.05- \\
32.13\end{array}$ & 2.32 & 39.86 & $\begin{array}{c}12.86 \pm \\
3.54\end{array}$ \\
\hline
\end{tabular}

Data are presented as mean \pm standard deviation (SD) ; SE : Standard error, Range : is the difference between the highest and lowest values in the set, $95 \% \mathrm{CI}$ : Confidence intervals (Lower and Upper); significance level is indicated by *, Where : $p>0.05: p$-value not significant, * indicates $\mathrm{p}<0.05$ (p-value significant) $; * *$ indicates $\mathrm{p}<0.01$ (p-value highly significant), in comparison with the corresponding control value.

disease, free fatty acids (FFA's) are released in large amounts followed by an elevated production of glucose, triglycerides (TG's) and very low-density lipoprotein (VLDL) (28). Also, FFA's decreased insulin sensitivity in muscles by discouraging insulin mediated glucose uptake. It is obvious that IR causes rising in blood glucose and FFA's levels, thus, worsening the IR and hyperglycemia with released FFA's further rises insulin secretion (hyperinsulinemia) forming a wicked circle (29). Furthermore, some environmental factors, like the obesity epidemic due to the physical exercise lack and increased caloric intake, are obviously responsible for the present elevation in the incidence of the metabolic syndrome worldwide (30). It has been speculated that TG's accumulation in skeletal muscles plays a lineal role in the aetiology of IR. So, chronic hyperglycemia and dyslipidemia in T2DM can both produce hurtful effects on $\beta$-cell structure and function $(31,32)$. Moreover, as DN progress, IR may be stimulating the decrease in renal function toward ESRD. High TG's levels are a risk factor for proteinuria development and TG-rich apolipoprotein B-containing lipoproteins clearly promote the progression of renal insufficiency (33).

Our data revealed that urea and creatinine levels were increased and GFR and $\mathrm{CrCl}$ levels were decreased in T2DM patients with and without DN when compared with controls (34).

Homocysteine (Hcy) is an amino acid created during the metabolism of methionine. The significant elevation in Hcy level in T2DM patients with and without $\mathrm{DN}$ in our study maybe due to IR. IR has been speculated to play a crucial role in the enhancement of atherosclerotic disease via dyslipidaemia, hypertension 
and hyperglycemia (35). Hence, hyperhomocysteinemia boosts IR by stimulating elevating glucose output, endoplasmic reticulum stress and upregulation phosphoenolpyruvate carboxykinase (PEPCK). This mechanism leads to increase Hcy levels in blood which may spend an atherothrombotic effect via elevating oxidative stress which may motivate endothelial dysfunction (11). Hcy levels in T2DM patients can be affected by both glomerular hyper- and hypofiltration which can respectively diminish and raise Hcy levels. Hcy is ultra-filtrated via the glomeruli and nearly fully reabsorbed in the tubules and degraded in renal tissue by transmethylation and transsulfuration. However, decreased GFR escorts MAU in the late phase of $\mathrm{DN}$, and decreasing of GFR causes increasing of Hcy levels (36).

Fructosamine (FA) is a ketoamine formed from the reaction between the carbonyl group of glucose and the amino group of protein. The significant increasing in FA level in T2DM patients with and without DN in our study could be due to protein losing states like diminished protein production, nephrotic syndrome, immunoglobulins, and physiologic or pathologic conditions associated to hypoproteinemia (12). It is possible that protein metabolism is modified in obese adults and could affected by inflammation. C-reactive protein (CRP) is known to be highly linked with BMI and is decreased by weight loss. There are other situations that may affect the interpretation of elevated FA levels include hyperuricemia, liver disease, acute illness and some infections (28). Moreover, hyperglycemia could consider as another possible cause for the significant increasing in FA level in T2DM patients with and without DN. The risk of secondary complications in patients with T2DM is highly connected to the chronic level of blood glucose. Nonenzymatic glycosylation is promoted in hyperglycemic conditions and chronic complications attached to DM. The products of nonenzymatic glycation combine in renal tissues and play a pivotal role in $\mathrm{DN}$ (37).

Cystatin C (CysC), a $13 \mathrm{kD}$ protein, is generated by a housekeeping gene in all nucleated cells. The significant increasing in CysC level in T2DM patients with and without DN could be due to advancement of CKD from stages I to III. Also, rise in serum CysC confront progression from normoalbuminuria to MAU and thereby suggestive a positive correlation between serum CysC and albumin creatinine ratio (ACR). So, serum CysC is a useful indicator in early detection of DN as it reflects decreasing in GFR as well as rise in ACR (1). Moreover, there are another possible and acceptable explanations for this significant elevation in CysC level in T2DM patients with and without DN such as IR and inflammation, and this may explain the connection between CysC and cardiovascular disease (CVD) in T2DM (5). The stabilized production rate of $\mathrm{Cys} C$ and its lack of excretion by the tubular epithelial cells may indicated that the kidney function is the main determinant of $\mathrm{Cys} C$ and even minimal kidney harm will result a significant increase in levels before the manifestation of traditional CKD markers (38). There is a chance of introducing CysC-based formulas without anthropometric variables to substitute creatinine-based equations in prediction GFR (39).

Selenium, a trace element, is a major component in glutathione peroxidase (GPx) which is one of the main antioxidant enzymes in the human body and responsible for preventing the production of free radicals, decreasing their activity or destroying them (19). Selenium levels were found significantly lower in T2DM patients with and without DN when compared to healthy control group. Selenium is known to work as an antioxidant and peroxynitrite scavenger when integrated into selenoproteins (20). This lack in Selenium levels may lead to oxidative stress, decline insulin secretion and elevate IR in some empirical models. Thereby, perhaps taking a causal function in the forward and pathogenesis of T2DM. Moreover, elevated oxidative stress and glycosylation play a main pathogenic role in diabetic endothelial cell dysfunc- tion in T2DM patients with and without DN (21).

Zinc is one of the fundamental trace elements which are involved in the synthesis, secretion, conformational integrity and storage of insulin. Our study reported that Zinc levels in T2DM patients with and without DN were lower than the controls. In the mammalian pancreas, Zinc is fundamental for the correct processing, secretion, storage and action of insulin in $\beta$-cells (22). Insulin is stored in secretory sacs or granules, where two $\mathrm{Zn}^{2+}$ ions coordinate six insulin monomers to generate the hexameric form on which maturated insulin crystals are based. It is also known that many other chronic disorders like DM could decrease Zinc levels, for example, DM increases the excretion of minerals such as Zinc in urine or may decrease gastrointestinal absorption of Zinc (40). Also, Hyperglycemia in DM is often linked with hyperzincuria, which is of kidney origin, and raise urinary loss of $\mathrm{Zn}^{2+}$ and declines of its level in entire body $\mathrm{Zn}^{2+}$. Kidney tubular flaw in handling Zinc and glucose-induced, osmotic diuresis are other probabilities. Furthermore, Zinc may enhance glycaemia and a restored Zinc status in patients with T2DM (with or without DN) may oppose the harmful effects of oxidative stress which helping to prevent complication beneficial antioxidant effects in people with T2DM (19). Zinc has been illustrated to have particularly importance in the light of the mischievous outcomes of oxidative stress in subjects with DM. Zinc has antioxidant properties, thus, it can balance macromolecules against radical stimulated oxidation (20).

Magnesium is a trace element which is responsible for maintaining some body functions. It is demanded for several enzymes' activities and for neuromuscular transmission. It plays a key role in glucose metabolism. The hypomagnesaemia in T2DM patients with and without DN in our study may be due to poor dietary intake, elevated urinary loss because of hyperglycemia, impaired absorption of $\mathrm{Mg}$, imperfect $\mathrm{Mg}$ reabsorption from kidney tubules, osmotic diuresis and loss of plasma protein bound to Magnesium (21). Magnesium diminution is said to decrease the insulin sensitivity, thereby, raising the risk of secondary complications. Hyperglycemia leads to decline cellular Mg levels. Hypomagnesaemia leads to decrease function of $\mathrm{Mg}$ dependent enzymes, collagen and ADP-induced platelet convenience and oxidative stress (22). Moreover, insulin lack and resistance lead to decreased tubular reabsorption of $\mathrm{Mg}$ and resulted hypomagnesemia prefer the onset and development of diabetic microangiopathy via a decreasing in activity of $\mathrm{Na}^{+} / \mathrm{K}^{+}$ATPase pump. So, hypomagnesemia alone foretells the progression to ESRD in patients with advanced DN (40).

Neutrophil Gelatinase-Associated Lipocalin (NGAL) is a small protein that belongs to the lipocalin proteins family secreted from neutrophils and several epithelial cell types including renal tubular cells. The significant elevation in NGAL level in T2DM patients with and without DN in our study could be due to acute kidney injury because NGAL is secreted by the kidney tubules and usually filtered by the glomerulus (14). In case of renal injury, the production of NGAL is elevated while the absorption is decreased by the renal tubules resulting a raising in NGAL levels (41). Furthermore, elevated albumin levels could play a pivotal role in raising levels of NGAL in T2DM patients with and without $\mathrm{DN}$ in our study because it activates kidney proximal tubular epithelial cells to stimulate tubulointerstitial inflammation (42). In contrast, high glucose levels and diabetic substrates (such as advanced glycation end-products, ultra-filtered growth factors and carbonyl intermediates) could excite a number of signalling pathways to enhance tubular cell hypertrophy and the interstitial precipitation of growth factors, cytokines, chemokines and adhesion molecules which are able to motivate additional inflammation and fibrosis as well as the stimuli that encourage epithelial damage which leading to increase NGAL 
levels (43).

8-Hydroxy-2-deoxyguanosine (8-OHdG) is one of the main products of DNA oxidation. Levels of 8-OHdG within a cell could considered as a measurement of oxidative stress. Production of ROS and lipid peroxidation are elevated in diabetic patients particularly in those with poor glycemic control. Oxidative stress may be important for evolution of diabetic vascular complications (15). So, Intracellular ROS can cause strand breaks in DNA and base modifications which leading to elevate levels of 8-OHdG. From the other hand, oxidative damage yields 8-OHdG after the manifestation of specified enzymatic cleavage when ROS stimulates 8-hydroxylation in mitochondrial and nuclear DNA (44). This progressive process clearly describes the connection between raised oxidative stress and T2DM and increased 8-OHdG secreted in urine synchronizes with high systemic DNA damage in patients with DN. Moreover, IR could play a pivotal role in producing oxidative stress, through free glucose activates aldose reductase activity and the polyol pathway, which could lead to decrease $\mathrm{NADPH} / \mathrm{NADP}^{+}$ratio (45). Increased intracellular glucose activates protein kinase $\mathrm{C}$ (PKC) via de novo synthesis of diacylglycerol. PKC stimulates oxidative stress by activating mitochondrial NADPH oxidase. So, the generated ROS may induce apoptosis, inflammation and endothelial dysfunction. Excess of FFA's, also can elevate oxidant production by $\beta$-oxidative phosphorylation through mitochondrial metabolism. These mechanisms may lead to increase 8 -OHdG levels significantly in T2DM patients with and without DN (46).

Based on our findings, we recommend that all T2DM patients should visit renal, optical, cardiovascular and neurological clinics regularly to take early steps to avoid and manage diabetic complications such as DN, diabetic retinopathy, CVD and diabetic neuropathy. Furthermore, the above investigated clinical and biochemical markers must be used in the hospitals and clinics of Basrah province in the future as new markers for early detection of the development of vascular complications in T2DM patients especially DN. Moreover, DN is a huge health issue that requires an appropriate treatment strategy. Therefore, the Iraqi state must understand this issue and show interest to allocate the essential resources to treat it. The socio-economic and medical support of patients (such as medical services, easier access to programs of health education, broader social support and higher insurance coverage) are crucial requirements for fixing this issue.

\section{CONCLUSION}

In conclusion, the results of this study demonstrate that obesity is a case accompanied by elevated levels of insulin and glucose which are the vigorous indicators for evaluation of the IR syndrome in diabetic patients especially with kidney disease. In addition, kidney disease is linked with the raising of IR, BMI levels in T2DM patients which had been observed via decreasing GFR, $\mathrm{CrCl}$ and increased urea, creatinine, Hcy levels. Furthermore, people who have T2DM (with or without $\mathrm{DN}$ ) tend to have high levels of Hcy, FA which give an indicator on the significant difference in oxidant/antioxidants status and may suggest a probable another role of oxidative stress mechanism in pathophysiology of T2DM which lead to DN. Additionally, CysC and creatinine were significantly higher in patients with $\mathrm{DN}$ compared to without DN. On the other hand, CysC may had a high significant diagnostic accuracy in distinguishing patients with DN than creatinine. Besides, the declining in some essential antioxidant trace elements levels (Selenium, Zinc and Magnesium) is a powerful indicator for evaluation of the oxidative stress syndrome in diabetic patients with and without $\mathrm{DN}$ than non-diabetics.
Moreover, urinary markers (Alb, NGAL and 8-OHdG) illustrated an excellent diagnostic value for early-stage of $\mathrm{DN}$ in patients with T2DM. Finally, Uncontrolled T2DM was associated with BMI, IR and physical activity in which the elevated levels of Hcy, FA, CysC, Alb, NGAL and 8-OHdG and decreased levels of trace elements may be used as early clinical markers of $\mathrm{DN}$.

\section{CONFLICT OF INTEREST}

All authors confirm that there are no conflicts of interest with people or organizations that could bias this report.

\section{ACKNOWLEDGEMENTS}

This research project was supported by the Ministry of Higher Education and Scientific Researches, University of Basrah, College of science, Department of chemistry, as part of the requirements for a master graduate program. The authors thank all of College of Nursing - University of Basrah and the staff of "Diabetes and Endocrine Glands Centre" at Al-Mawany teaching hospital in Basrah province-Iraq for providing their kind support and facilities to accomplish the present research project within time.

\section{REFERENCES}

1. Kumar ASA, Kumar ASA : Serum cystatin C and serum creatinine levels in type 2 diabetes mellitus. Int J Res Med Sci 3(1) : 174-177, 2015

2. Donovan EL, Buckels EJ, Hancock S, Smeitink D, Oliver $\mathrm{MH}$, Bloomfield FH, Jaquiery A : Twin conception in sheep leads to impaired insulin sensitivity and sexually dimorphic adipose tissue and skeletal muscle phenotypes in adulthood. Reprod Sci 24(6) : 865-881, 2017

3. Elebrashy IN, El Meligi A, Rashed L, Salam RF, Youseef E, Fathy SA : Thyroid dysfunction among type 2 diabetic female Egyptian subjects. Ther Clin Risk Manag 12 : 1757 1762,2016

4. Kopel J, Pena-Hernandez C, Nugent K: Evolving spectrum of diabetic nephropathy. World J Diabetes 10(5) : 269-279, 2019

5. Bashier AM, Fadlallah AAS, Alhashemi N, Thadani PM, Abdelgadir E, Rashid F : Cystatin C and its role in patients with type 1 and type 2 diabetes mellitus. Advances in Endocrinology $11: 1-8,2015$

6. Macisaac RJ, Ekinci EI, Jerums G : Markers of and risk factors for the development and progression of diabetic kidney disease. American Journal of Kidney Diseases 63(2) : S39S62, 2014

7. Narres M, Claessen H, Droste S, Kvitkina T, Koch M, Kuss $\mathrm{O}$, Icks A: The incidence of end-stage renal disease in the diabetic (compared to the nondiabetic) population: A systematic review. PLoS ONE 11(1) : 1-28, 2016

8. Sekulic SP, Sekulic M : Rheological influence upon the glomerular podocyte and resultant mechanotransduction. Kidney Blood Press Res 40(2) : 176-187, 2015

9. Kim MR, Yu SA, Kim MY, Choi KM, Kim CW : Analysis of glycated serum proteins in type 2 diabetes patients with nephropathy. Biotechnol Bioproc Engin 19(1) : 83-92, 2014

10. Gluhovschi C, Gluhovschi G, Petrica L, Timar R, Velciov $\mathrm{S}$ : Urinary biomarkers in the assessment of early diabetic nephropathy. Journal of Diabetes Research 2016 : 1-13, 2016 
11. Shin C, Baik I : Leukocyte telomere length is associated with serum vitamin B12 and homocysteine levels in older adults with the presence of systemic inflammation. Clin Nutr Res 5(1) : 7-14, 2016

12. Selvin E, Warren B, He X, Sacks DB, Saenger AK: Establishment of community-based reference intervals for fructosamine, glycated albumin, and 1, 5-anhydroglucitol. Clin Chem 64(5) : 843-850, 2018

13. Upadhyay AK, Sowdhamini R : Genome-wide prediction and analysis of $3 \mathrm{D}$-domain swapped proteins in the human genome from sequence information. PLOS ONE 11(7) : 1-20, 2016

14. AL-Mosawy AN, Hatroosh SJ : Evaluation of effectiveness of Myrrh gum extract on some biochemical and histological parameters in male rats induced chronic renal failure (CRF). Plant Archives 19(2) : 1711-1717, 2019

15. Hojs R, Ekart R, Bevc S, Hojs N : Biomarkers of renal disease and progression in patients with diabetes. J Clin Med 4(5) : 1010-24, 2015

16. ADA : American diabetes association. Diabetes care. Introduction: Standards of medical care in diabetes 41(Supplement 1) : S1-S2, 2018

17. Jerums G, Ekinci E, Panagiotopoulos S, MacIsaac RJ : Early glomerular filtration rate loss as a marker of diabetic nephropathy. European Endocrinology 8(1) : 27-31, 2012

18. Zanetti D, Bergman H, Burgess S, Assimes TL, Bhalla V, Ingelsson $\mathrm{E}$ : Urinary albumin, sodium, and potassium and cardiovascular outcomes in the UK biobank : Observational and mendelian randomization analyses. Hypertension 75(3) : 714-722, 2020

19. Al-Fartosy AJM, Awad NA, Mahmood RA : A comparative study of leptin, oxidant/antioxidant status and some trace elements in women of healthy control and unexplained infertility in Basrah-Iraq. Indones Biomed J 11(3) : 327-337, 2019

20. Al-Fartosy AJM, Awad NA, Abdalemam DJ : Biochemical study of the effect of insulin resistance on adiponectin, lipid profile and some antioxidants elements with relation to obesity in type 2 diabetic patients/Basrah-Iraq. Amer J Biochem $7: 73-82,2017$

21. Al-Fartosy AJM, Awad NA, Mohammed AH : Intelectin-1 and endocrinological parameters in women with polycystic ovary syndrome : Effect of insulin resistance. Ewha Med J 43(1) : 1-11, 2020

22. Al-Fartosy AJM, Awad NA, Alsalimi SA: Osteoprotegerin and some trace elements in type 2 diabetic patients with or without nephropathy : Effect of insulin resistance. International Medical Journal 25(4) : 1771-1784, 2020

23. Chen M, Xia J, Pei G, Zhang Y, Wu S, Qin Y, Deng Y, Guo S, Guo Y, Xu G, Han M : A more accurate method acquirement by a comparison of the prediction equations for estimating glomerular filtration rate in Chinese patients with obstructive nephropathy. BMC Nephrology $17: 1-8,2016$

24. Park SK, Ryoo JH, Oh CM, Choi JM, Jung JY : Longitudinally evaluated the relationship between body fat percentage and the risk for type 2 diabetes mellitus : Korean genome and epidemiology study (KoGES). European Journal of Endocrinology 178(5) : 513-521, 2018

25. Boughton CK, Munro N, Whyte M : Targeting beta-cell preservation in the management of type 2 diabetes. The British Journal of Diabetes 17(4) : 134-144, 2017

26. Wang S, Ma W, Yuan Z, Wang S, Yi X, Jia H, Xue F : Association between obesity indices and type 2 diabetes mellitus among middle-aged and elderly people in Jinan, China : A cross-sectional study. BMJ Open 6 : 1-9, 2016
27. Budiyani L, Purnamasari D, Simadibrata M, Abdullah $\mathrm{M}$ : Differences in the insulin resistance levels measured by HOMA-IR between patients with erosive and non-erosive gastroesophageal reflux disease. Journal of the ASEAN Federation of Endocrine Societies 32(2) : 139-144, 2017

28. Al-Fartosy AJM, Mohammed IM : Comparison of insulin resistance, prolactin and $\mathrm{HbA1c}$ with relation to obesity in men and women of healthy control and diabetic patients/Meisan-Iraq. International Journal of Current Research 9(8) : 55643-55648, 2017

29. Cicero AFG, Rosticci M, Parini A, Morbini M, Urso R, Grandi E, Borghi C : Short-term effects of a combined nutraceutical of insulin-sensitivity, lipid level and indexes of liver steatosis : A double-blind, randomized, cross-over clinical trial. Nutr J 14 : 1-6, 2015

30. Xin YL, Wang Y, Chi J, Zhu X, Zhao H, Zhao S, Wang Y : Elevated free fatty acid level is associated with insulin-resistant state in nondiabetic Chinese people. Diabetes Metab Syndr Obes $12: 139-147,2019$

31. Febbraio $\mathrm{M}:$ Health benefits of exercise-more than meets the eye!. Nat Rev Endocrinol 13(2) : 72-74, 2017

32. Tangvarasittichai $\mathrm{S}$ : Oxidative stress, insulin resistance, dyslipidemia and type 2 diabetes mellitus. World J Diabetes 6(3) : 456-480, 2015

33. Mahfouz MH, Assiri AM, Mukhtar MH : Assessment of neutrophil gelatinase-associated lipocalin (NGAL) and retinol-binding protein 4 (RBP4) in type 2 diabetic patients with nephropathy. Biomarker Insights 11 : 31-40, 2016

34. Sirivole MR, Eturi S : A study on blood urea and serum creatinine in diabetes mellitus from Sangareddy district, Telangana, India. International Journal of Medical and Health Research 3(12) : 132-136, 2017

35. Platt DE, Hariri E, Salameh P, Merhi M, Sabbah N, Helou M, Mouzaya F, Nemer R, Al-Sarraj Y, El-Shanti H, Abchee AB, Zalloua PA : Type II diabetes mellitus and hyperhomocysteinemia : A complex interaction. Diabetol Metab Syndr $9: 1-7,2017$

36. Aouda MA, Hamza AS : The impact of smoking and diabetes on homocysteine levels in patients with myocardial infarction. J.Thi-Qar Sci 6(2) : 75-80, 2017

37. Neelofar K, Ahmad $\mathrm{J}$ : Glycosylation gap in patients with diabetes with chronic kidney disease and healthy participants : A comparative study. Indian J Endocr Metab 21(3) : 410-414, 2017

38. Dandana A, Gammoudi I, Chalghoum A, Chahed H, Addad F, Ferchichi S, Miled A : Clinical utility of serum cystatin $\mathrm{C}$ in predicting coronary artery disease in patients without chronic kidney disease. Journal of Clinical Laboratory Analysis 28(3) : 191-197, 2014

39. Ajith $\mathrm{M}$ : Appraisal of diabetic nephropathy through GFR estimation in view of various cystatin $\mathrm{C}$ equations : A relative investigation. Indian Journal of Applied Research 9(7) : 40-43, 2019

40. Al-Fartosy AJM, Shanan SK, Awad NA : Biochemical study of the effects of some heavy metals on oxidant/antioxidant status in gasoline station workers/Basra-Iraq. International Journal of Scientific and Research Publications 7(2) : 83-94, 2017

41. Siddiqui K, Al-Malki B, George TP, Nawaz SS, Al Rubeaan $\mathrm{K}$ : Urinary $\mathrm{N}$-acetyl-beta-d-glucosaminidase (NAG) with neutrophil gelatinase-associated lipocalin (NGAL) improves the diagnostic value for proximal tubule damage in diabetic kidney disease. 3 Biotech $9: 1-7,2019$

42. Zeng XF, Lu DX, Li JM, Tan Y, Li Z, Zhou L, Xi ZQ, Zhang SM, Duan W : Performance of urinary neutrophil gelatinase-associated lipocalin, clusterin, and cystatin $\mathrm{C}$ in 
predicting diabetic kidney disease and diabetic microalbuminuria: A consecutive cohort study. BMC Nephrology 18 (1) : 1-10, 2017

43. Hong DSC, Oh IH, Park JS, Lee CH, Kang CM, Kim $\mathrm{GH}$ : Evaluation of Urinary Indices for Albuminuria and Proteinuria in Patients with Chronic Kidney Disease. Kidney Blood Press Res 41 : 258-266, 2016

44. Ye X, Jiang R, Zhang Q, Wang R, Yang C, Ma J, Du H : Increased 8-hydroxy-2'- deoxyguanosine in leukocyte DNA from patients with type 2 diabetes and microangiopathy.
Journal of International Medical Research 44(3) : 472-482, 2016

45. Chou ST, Tseng ST : Oxidative stress markers in type 2 diabetes patients with diabetic nephropathy. Clin Exp Nephrol 21(2) : 283-292, 2016

46. Kong L, Wang Y, Luo M, Tan Y, Cui W, Miao L: Prevention of streptozotocin-induced diabetic nephropathy by MG132 : Possible roles of Nrf2 and IкB. Oxidative Medicine and Cellular Longevity 2017 : 1-12, 2017 